\title{
The Effect of Working Memory Training on Children with Reading Disabilities
}

\begin{abstract}
Yuexin Ren*
Bishop Monogorty high school

*Corresponding author. Email: yren@student-bmhs-la.org

ABSTRACT

Working memory training is intended to improve a person's working memory.In this paper, the effects of working memory training on children with learning disabilities are investigates. For better illustration, the definitions of working memory and the relationship between working memory and dyslexia are introduced. Besides, the training process and measures help dyslexia people are presented. Based on the analysis, working memory training for dyslexia can not only improve performance of skills, but can also improve reading skills, showing an effect of training migration in reading speed tasks and reading comprehension tasks. However, these effects need further verification from direct empirical studies on this aspect. These results shed light for ......
\end{abstract}

Keywords: Working memory, children, dyslexic.

\section{INTRODUCTION}

Dyslexia is divided into acquired dyslexia and developmental dyslexia. Acquired dyslexia refers to reading difficulties caused by congenital or acquired brain damage and corresponding visual and auditory impairments. Developmental dyslexia refers to the phenomenon that children with normal intelligence have no obvious neurological or organic damage in the development process, but their reading level is significantly behind their corresponding intelligence level or biological age. The concept of working memory was put forward by Baddeley [1] and continuously improved by some famous psychologists and cognitive neuroscientists. Its function is to process and store information temporarily. In other words, the working memory should not only store the information for a short time, but also process it and call the information of the long-term memory temporarily.

Dyslexia is simply a reading and spelling disorder caused by the brain's inability to process visual and auditory information in a coordinated way. Paying special attention to distinguishing it from dyslexia, which is caused by mental retardation, as opposed to the fact that many people suffer from extremely high IQs, including geniuses. Leonardo da Vinci, Thomas Edison, Albert Einstein and John F. Kennedy were all considered "stupid kids" who got poor grades in childhood, and scientists have found that they are typical examples of dyslexia. Its characteristics are mainly reflected in the aspect of literacy and reading. The origination reasons are complex and multi-faceted, but it can be scientifically corrected in children.

In this paper, the effect of working memory training on children with reading disabilities will be investigated.

\section{RELATIONSHIP BETWEEN WORKING MEMORY AND DYSLEXIA}

\subsection{Detailed introduction to working memory}

Working memory is a system on our brain, which is also a cognitive system with a limited capacity that can hold information temporarily. Working memory is important for reasoning as well as the guidance of decision-making and behavior. Besides, it is often used as synonymously with short-term memory short term memory. However, some theorists consider the two forms of memory distinct, deeming that working memory allows for the manipulation of stored information, while short-term memory only refers to the short-term storage of information. Working memory is a theoretical concept central to cognitive psychology, neuropsychology, and neuroscience. 


\subsection{Dyslexia and its manifestations}

Human begins will have some special behavior if they have dyslexia. Dyslexic children may be physically and socially immature in comparison to their peers. This can lead to a poor self-image and less peer acceptance. Dyslexics' social immaturity may make them awkward in social situations. Many dyslexics have difficulty reading social cues. Those children will become fearful because of their constant frustration and confusion in school. In the past, researchers have done a lot of research on the performance of dyslexia and its harm to children's development, in order to provide any meaningful help for people with dyslexia. Among dyslexic readers, the most persistent working memory deficit is the storage capacity of phonological short-term memory.

\subsection{Relationship between the two}

The relationship between working memory and children's dyslexia is very important. Readers with dyslexia cannot remember large amounts of sequential, verbal information (e.g., letters, phonemes, words and phrases), as the average reader can. Many educators and psychologists acknowledge that people with learning disabilities are likely to have deficits in one or more cognitive processes, including speech processing, auditory processing, long-term extraction, attention, short-term memory, and working memory. Swanson et al. [2] computed 578 effective cases in a meta study and found that children with reading disability were distinctively disadvantaged compared with average readers. Specifically, it reflects in short term memory measures requiring the recall of phonemes and digit sequences and working memory measures requiring the simultaneous processing and storage of digits within sentence sequences and final words from unrelated sentences. No significant moderating effects emerged for reading level on memory ESs. Researchers have consistently found that children with a variety of learning disabilities show poor working memory performance, especially in verbal and executive working memory. When children with learning disabilities were paired with a control group of children with the same IQ, the learning disability group showed deficits in certain aspects of working memory within the child.

\section{WORKING MEMORY TRAINING PARADIGM}

One example is the CogMed working memory training program, which is owned and distributed by the Pearson publishing group. CogMed working memory training includes multiple computerized verbal and visuospatial memory span tasks that trainees practice several times per week for several weeks. Another example of working memory tasks could include holding a person's address in mind while listening to other instructions (e.g., road descriptions, stories) [3]. Other research is of all the cognitive training studies to date, the fast majority focus on working memory as the primary target for remediation [4]. Working memory is the cognitive system responsible for storing, integrating and manipulating information during complex and demanding activities [1]. Working memory measures. digit, word list, listening, and block recall (inspired by Corsi) [5] of the Working Memory Test Battery for Children (WMTB-C, [6]) were used [7]. In addition, in line with St. Clair-Thompson et al. [8], all children were tested with the spatial span, an adapted version of the Automated Working Memory Assessment (AWMA) [9]. Finally, backward word list recall and backward block recall were utilized [10]. All span tasks were based on Baddeley's working memory model [11]. Maximum span length was nine for digit and block recall; seven for word list, backward digit, and backward block recall; and six for listening recall and spatial span. Sequence of onset was two for backward digit recall, backward word recall, and backward block recall and one for all other tasks. In accordance with the WMTB-C, [12] each length series consisted of six trials.

\section{EFFECT OF WORKING MEMORY TRAINING ON DYSLEXIA}

Working memory training can help dyslexia. For instance, students with dyslexia have strengths in visualspatial working memory. Studies comparing visual memory for novel objects confirm that students with dyslexia perform similarly to normal readers. Their good visual working memory means that they learn words as a unit, rather than work out their individual sounds. There is also some difference between now and the past. Contemporarily, the Dyslexia, or reading disability, is a disorder of the language processing systems in the brain. Imaging research confirmed that instruction in the alphabetic principle caused distinct differences in brain activation patterns in the students with RD [13].

For example, students with dyslexia have an advantage in visuospatial working memory. Studies comparing visual memories of novel objects have confirmed that dyslexia students perform similarly to normal readers. Their good visual working memory indicates they learn words as a group, rather than pronouncing them individually. There is no known way to be sure that abnormal brain behaviour causes the disease. This is a lifelong problem. The most important thing is to monitor the condition on a daily basis for treatment, rather than waiting for symptoms to appear before a larger treatment, which can waste a lot of valuable time. The most effective way to treat dyslexia is to detect it early. Plenty of current cognitive training programs (e.g., Klingberg's CogMed or Prins' Braingame Brian) [3] are based on the assumption that participants themselves will elaborate on the different strategies that could improve their performance by repeatedly performing WM tasks. 


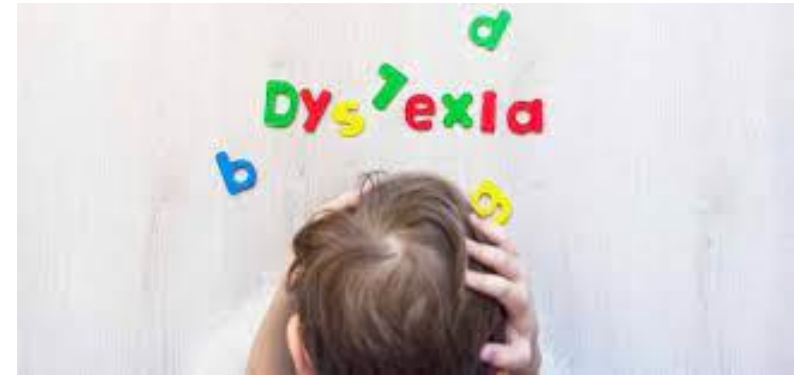

Figure 1. How can I help my child with Dyslexia

\section{CONCLUSION}

In summary, research has shown that WM training for dyslexia can not only improve performance of training skills, but can also improve reading skills, showing an effect of training migration in reading speed tasks and reading comprehension tasks. Learning disabilities are a constantly changing field. With the advent of technologies that allow scholars to study the brain in action, one will not only understand the normal reading process, but also understand the condition that the system is not working. It is hoped that one will be able to prevent learning disabilities, or at least be able to develop innovative and successful interventions. It is also hoped that we will be more adept at identifying older children to prevent certain emotional and social difficulties related to learning disabilities. So far, neuroscience and genetics are expected to become new avenues in our study of learning disabilities. Families with a history of learning disabilities need further research to provide them with appropriate support and assist in early intervention. Schools are becoming more and more adept at dealing with children with different types of learning disabilities. Besides, the ability to properly evaluate children from ethnic minorities will also improve. These results offer a guideline for ......

\section{REFERENCES}

[1] Baddeley, A. D., \& Hitch, G. J. (2000). Development of working memory: Should the Pascual-Leone and the Baddeley and Hitch models be merged?. Journal of experimental child psychology, 77(2), 128-137.

[2] Swanson, H. L., Zheng, X., \& Jerman, O. (2009). Working memory, short-term memory, and reading disabilities: A selective meta-analysis of the literature. Journal of learning disabilities, 42(3), 260-287.

[3] Klingberg, T . Fernell, E. Olesen, P J Johnson,M. Gustafsson, P. Dahlstrom K et al. (2005) Computerized training of working memory in children with ADHD-a randomized, controlled trial. Journal of the American Academy of Child and Adolescent Psychiatry, 44(2), 177-186.
[4] Rapport, M. D., Orban, S. A., Kofler, M. J., \& Friedman, L. M. (2013). Do programs designed to train working memory, other executive functions, and attention benefit children with ADHD? A metaanalytic review of cognitive, academic, and behavioral outcomes. Clinical Psychology Review, 33(8), 1237-1252.

[5] Corsi, P. (1972). Memory and the medial temporal region of the brain. Unpublished doctoral dissertation), McGill University, Montreal, QB.

[6] Pickering, S. J., \& Gathercole, S. E. (2001). Working memory test battery for children (WMTB-C). London, UK: Pearson.

[7] Geary, David C., Mary K. Hoard, Jennifer ByrdCraven, Lara Nugent, and Chattavee Numtee. Cognitive mechanisms underlying achievement deficits in children with mathematical learning disability. Child development 78, no. 4 (2007): 1343-1359.

[8] St Clair-Thompson, Helen L., and Susan E. Gathercole. Executive functions and achievements in school: Shifting, updating, inhibition, and working memory. Quarterly journal of experimental psychology 59, no. 4 (2006): 745-759.

[9] Alloway, T. P. (2007). Working memory, reading, and mathematical skills in children with developmental coordination disorder. Journal of experimental child psychology, 96(1), 20-36.

[10] Passolunghi, M. C., \& Mammarella, I. C. (2010). Spatial and visual working memory ability in children with difficulties in arithmetic word problem solving. European Journal of Cognitive Psychology, 22(6), 944-963.

[11] Baddeley, A., Logie, R., Bressi, S., Sala, S. D., \& Spinnler, H. (1986). Dementia and working memory. The Quarterly Journal of Experimental Psychology Section A, 38(4), 603-618.

[12] de Weerdt, F., Desoete, A., \& Roeyers, H. (2013). Working memory in children with reading disabilities and/or mathematical disabilities. Journal of learning disabilities, 46(5), 461-472.

[13] Peijnenborgh, J. C., Hurks, P. M., Aldenkamp, A. P., Vles, J. S., \& Hendriksen, J. G. (2016). Efficacy of working memory training in children and adolescents with learning disabilities: A review study and meta-analysis. Neuropsychological rehabilitation, 26(5-6), 645-672. 